\title{
On the Accuracy of Compositional Quantification for Atom Probe Tomography
}

\author{
Mattias Thuvander ${ }^{1}$ \\ ${ }^{1 .}$ Department of Physics, Chalmers University of Technology, Gothenburg, Sweden
}

Atom probe tomography (APT) is a versatile technique for characterization of materials with a sub-nm resolution possessing exceptional possibilities for measuring composition on a very small scale, in three dimensions. As the method relies on detection of individual ions that have been field evaporated from a needle-shaped sample, quantification is based on counting atoms. Therefore, no calibration factors are involved and the sensitivity is the same for all elements (with the exception of hydrogen, where ions originating from the sample cannot be distinguished from ions originating from the vacuum). The accuracy of compositional measurements is generally very high, and often the errors given are very small. For example an error of 0.01 at.\% is common, suggesting that the relative error for an element of concentration $10 \%$ is as small as $10^{-3}$. Here, a few factors that may deteriorate the accuracy are discussed.

The resulting mass spectrum from an APT analysis presents the number of ions detected as a function of mass-to-charge ratio. Usually, the assignment of the dominating mass spectrum peaks is straight forward (with well-known overlaps such as ${ }^{28} \mathrm{Si}^{2+}$ and ${ }^{14} \mathrm{~N}^{+}$). There are now even automated approaches for assigning mass spectrum peaks [1].

For small, uncomplicated datasets the accuracy is mainly determined by the counting statistics, and, in particular for minor peaks, by the background (noise) subtraction (including the counting statistics of the background subtraction). However, for large datasets, containing several million atoms, the errors related to the counting statistics become very small, and it is likely that the accuracy is determined by other factors. One such factor is the dependence of the concentration with crystallographic direction, making the concentration vary in the $\mathrm{x}-\mathrm{y}$ plane. As an example, the frequency distribution of the $\mathrm{Cr}$ concentration in a presumably random Ni-base alloy was constructed from the atoms inside a cylinder in the z-direction, for various cylinder radii (i.e. using different parts of the x-y plane). The reduced $X^{2}$ values obtained from comparison with the expected binomial distribution are shown in figure 1 . The result shows that the material appears to be more non-random (values $>1$ ) as the radius of the cylinder increases, clearly an effect of un-real concentration variations in the x-y plane. In a second example, using a dataset from another solution annealed Ni-base alloy, the $\mathrm{Cr}$ concentration was determined in nine sub-volumes oriented in the z-direction, as shown in figure 1 . The standard deviation was 0.62 at. $\%$, compared to the value of 0.07 at.\% considering the counting statistics alone.

In cases when peak overlaps can be deconvoluted using the natural abundance of different isotopes, the accuracy decreases both due to the statistics of the deconvolution and the spread in the natural abundance itself, which is substantial for several elements, including B and C [2]. A special type of overlap occurs when pairs like $\mathrm{X}^{+}$and $\mathrm{X}_{2}{ }^{2+}, \mathrm{X}_{2}{ }^{+}$and $\mathrm{X}_{4}{ }^{2+}$, etc., are present. If the element has more than one isotope the overlap can be deconvoluted, as for the case of $\mathrm{Mo}^{+}$and $\mathrm{Mo}_{2}{ }^{2+}$ in figure 2 , or the commonly observed $\mathrm{C}_{2}{ }^{+}$and $\mathrm{C}_{4}{ }^{2+}$ overlap. However, the accuracy is affected negatively. If the element, on the other hand, only has one isotope (like $\mathrm{Al}, \mathrm{Nb}, \mathrm{P}, \mathrm{As}$ and $\mathrm{Mn}$ ), such overlaps cannot be noticed, with the risk of a severe increase of the error. The situation would clearly benefit from having kinetic energy sensitive detectors. 
Other effects that might reduce the accuracy include the presence of ions originating from the vacuum, detector pile-up due to multiple events [3], preferential evaporation between pulses, formation of neutrals, dissociation of molecular ions [4], improper choice of mass range widths, as well as changes to the material during sample preparation. As some of these effects might be very significant, and at the same time hard to track down in a single analysis, it is important to perform tests of the influence of the analysis parameters on the measured composition to assure that the accuracy is as high as possible. Generally, it makes more sense to use the standard deviation from several measurements, or the standard deviation obtained using sub-volumes, than to use the error from the counting statistics.

\section{References:}

[1] D Haley et al, Ultramicroscopy 159 (2015), p. 338.

[2] M Berglund and ME Wieser, Pure Appl. Chem. 83 (2011), p. 397.

[3] M Thuvander et al, Ultramicroscopy 111 (2011), p. 604.

[4] DW Saxey, Ultramicroscopy 111 (2011), p. 473.
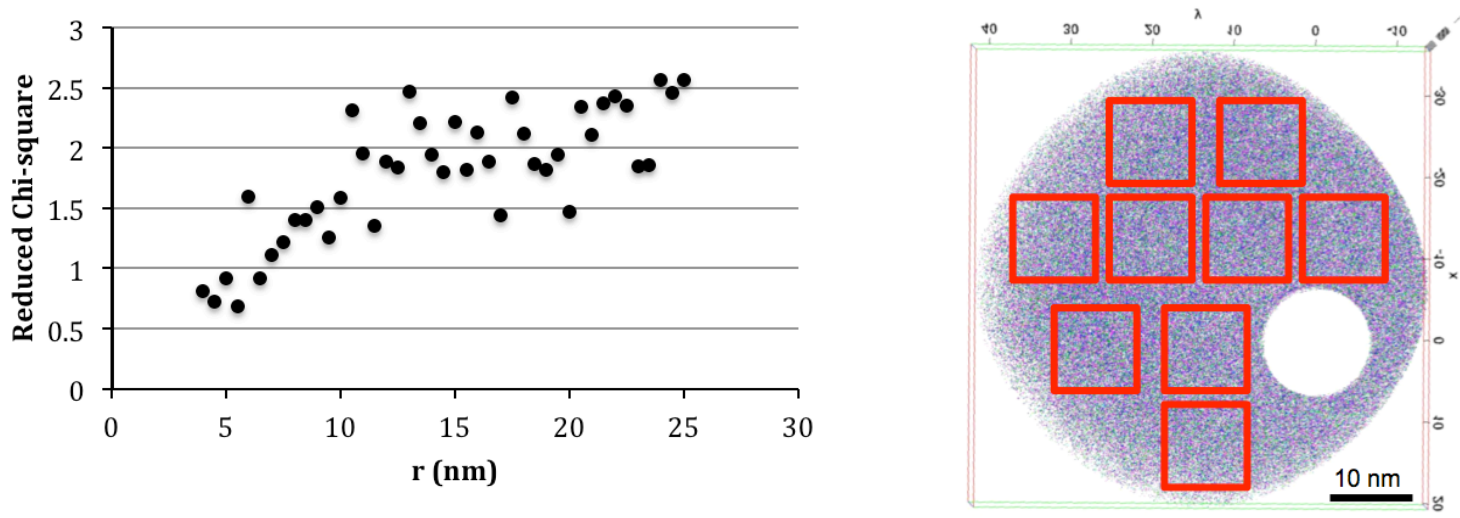

Figure 1. Reduced Chi-square values of the Cr frequency distribution as function of the radius of the selected cylinder (voltage pulse analysis of a Haynes alloy), left. Reconstructed volume from analysis of Alloy 690 (voltage pulsing) viewed along the direction of analysis, right. For estimating the accuracy, the composition was measured in nine sub-volumes of size $12 \times 12 \times 65 \mathrm{~nm}^{3}$. The atoms close to a pole have been removed because the Cr concentration there was clearly too high.

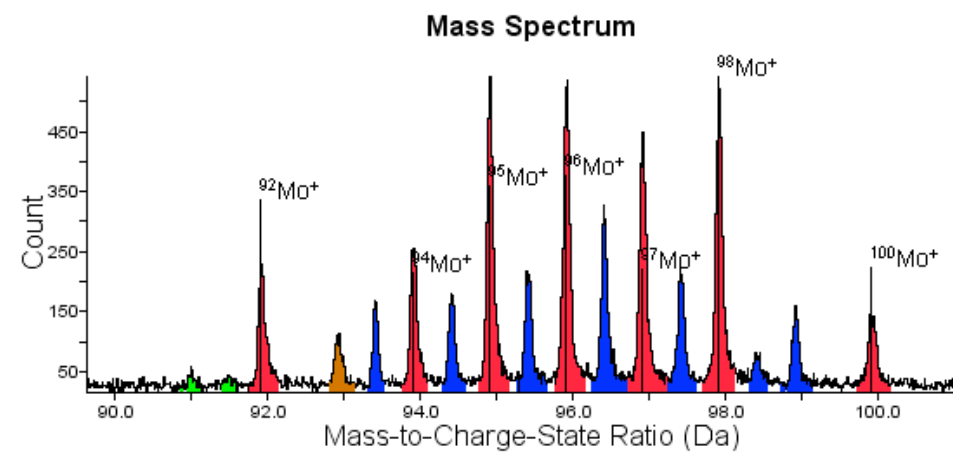

Figure 2. Mass spectrum showing overlaps between $\mathrm{Mo}^{+}$and $\mathrm{Mo}_{2}{ }^{2+}$ from analysis (green laser $0.3 \mathrm{~nJ}$ ) of a stainless steel. The small peak at $93 \mathrm{Da}$ is probably an overlap between $\mathrm{Nb}^{+}$and ${ }^{92} \mathrm{Mo}^{94} \mathrm{Mo}^{2+}$, but a fraction of the ions might as well be $\mathrm{Nb}_{2}{ }^{2+}$. 